$\mathrm{k}$ eine $\mathrm{r}$ W ise hat jedoch die vorliegende Mitteilung des Herrn Dr. M. einschliefslich des Untersuchungs-Berichtes aus dem 2. chemischen Institut der hiesigen Universität zur Aufklärung dieser Affaire beigetragen.

\title{
Ueber den Nachweis der Digitalis-Glycoside und ihrer Spaltungsprodukte durch eisenhaltige Schwefelsäure.
}

\author{
Von H. Kiliani. \\ (Eingegangen den 11. III. 1896.)
}

Dafs einzelne Digitalis-Glycoside beim Auflösen in konz. Schwefelsäure und darauffolgendem Zusatze von Oxydationsmitteln wie Brom, Salpetersäure, Eisenchlorid gewisse Farbenreaktionen liefern, ist schon lange bekannt; solche Angaben finden sich z. B. auch in der oft citierten Abhandlung Schmiedeberg's. Alle einschlägigen älteren Vorschriften beschränken sich aber daraut, der Zusatz "tines Tropfens" verdünnten Eisenchlorids etc. zu enpfehlen, was in diesem Falle nicht genügt, weil hier sowohl die Intensität als namentlich auch die Dauer der Farbenerscheinung in besonders hohem Grade abhängig ist von der Menge des angewandten Oxydationsmittels.

In einer Abhandlung über Digitalinum verum¹) habe jch früher berichtet, dal's man bei diesem Glycoside und beim Digitaligenin eine sehr hübsche und auffallend beständige Farbenreaktion erhält, wenn jene Körper einfäach in englischer Schwefelsäure ohne jeden weiteren Zusatz gelöst werden, wobei ich vermutete, dafs die Erscheinung auf den, fast nie fehlenden, geringen Gehalt der Säure an Stickstolfverbindungen zurückzuführen sein dürfte. Später mathte ich aber die Beobachtung, dals die verschiedenen englischen Süuren des Handels sich nicht gleichmälsig zur Anstellung jener Versuche eignen. Als ich nun vor einigen Monaten zufällig in den Besitz einer Säure kam, wolche ganz vorzüglich in dein gewünschten Sinne reagierte, veranlafste ich Herrn Assistent Dr. Munkert zu einer genaneren

1) Dieses Archiv 230, S. 254.

Arch. d. Pharm. Cexxxiv. Bds. 4. Iett. 
Untersuchung derselben. Hierbei wurde eine verschwindend geringe Menge von Säuren des Stickstoffs gefunden, wohl aber ein relativ beträchtlicher Gehalt an Eisensalz. Herr Dr. Munkert machte sodann verschiedene Mischungen von reiner konz. Schwefelsäure und schwefelsaurem Eisenoxyd und fand, dals man ein tadelloses und absolut sicheres Reagens a f dieverschiedenen Digi talisstoffe erhält, wenn man $100 \mathrm{ccm}$ reiner konz. Schwefelsäure vermischt mit $1 \mathrm{ccm}$ einer Ferrisulfatlösung, welchedurch A aflösen von 5 g käutlichem Ferrum sulfuric. oxydat. pur. ${ }^{1}$ ) in $100 \mathrm{ccm}$ Wasser gewonnen wurde.

Mittelst dieser Schwefelsäure, welche den Vorzug besitzt, dafs ihr Eisengehalt wenigstens annähernd ein bestimmter ist, lassen sich nun Digitalinum verum, Digitoxin und Digitonin sowie deren Spaltungsprodukte, wenn sie in annähernd reinem Zustande anch nur in kleinster Menge vorliegen, äufserst scharf erkennen und unterscheiden: Man übergiefst zu diesem Zwecke einige Stäubchen der zu prüfenden Substanz in einem Proberöhrchen mit 4-5 ccm obiger Säure und bringt die ursprünglich schon vorhandenen Körnchen oder die unter dem Einflusse der Säure gebildeten Klümpchen (letztere namentlich beim Digitonin) mit Hülfe eines feinen Glasstabes zu gleichmärsiger Verteilung und Auflösung. Hiebei werden folgende Erscheinungen beobachtet:

Digitali n u m ver u m tärbt sich im ersten Momente intensiv goldgelb und löst sich dann mit roter Farbe. Diese geht jedoch rasch in ein prachtvolles und tagelang beständiges Rotviolett über. Wurde etwas zu viel Glycosid genommen, so bleibt die ganze Lösung rot; schtittelt man aber in diesem Falle, so zeigen jedenfalls die dünneren Schichten, welche hiedurch an der Oberfläche der Flüssigkeit entstehen, die schöne rotviolette Farbe, welche ziemlich identisch sein dürfte mit jener der Digitalisblüte.

Das Digitaligen in liefert die gleichen Farbenerscheinungen, nur mit gröfserer Intensität, d. h. die Reaktion läfst sich mit noch geringeren Mengen Substanz durchführen.

1) Unser Präparat enthielt 53,5 Proz. $\mathrm{Fe}_{2}\left(\mathrm{SO}_{4}\right)_{3}, 24,9$ Proz. $\mathrm{H}_{3} \mathrm{O}$ und aufserdem treie Schwefelsäure. 
Digitoxin wird beim Uebergiefsen mit dem Reagens sofort ganz dunkel, als ob vollständige Verkohlung einträte; dann entsteht eine klare, schmutzig braunrote Lösung.

Digitoxigenin zeigt jene Dunkelfärbung nicht mehr; die Säure wird hier langsam eigenartig rot und entwickelt dabei eine auffallend starke Fluorescenz.

Digitonin und Digitogenin endlich verursachen, wenn sie in ebenso geringer Quantität zur Anwendung gelangen, wie die vorher besprochenen Substanzen, nicht die geringste Färbung der Säure, und gelbst mit der 3-4 fachen Menge Material orhält man böchstens einen ganz schwachen, gelblichen Farbenton.

Von den angeführten Reaktionen ist nun am wenigsten charakteristisch jene des Digitoxins, denn ein schmutziges Braunrot können auch andere Substanzen beim Zusammentreffen mit konz. Sehwefelsäure hervorrufen. Gläcklicherweise lälst sich jedoch diese Lücke leicht ausfüllen durch Kombination obiger Methode mit derjenigen von Keller'). Letzterer schreibt, man solle die Glycoside in Eisessig lösen, dazu "ein Tröpfchen" Eisenchlorid geben und dann reine konz. Schwefelsüure vorsichtig darunter schichten. Bei Gegenwart von Digitoxin bildet sich an der Grenzschichte eine dunkle Zone und über dieser, also im Eisessig, ein tiefblaues Band.

Diese Angabe Keller's ist ganz richtig, doch gelangt bei Einhaltung seiner Vorschrift die blaue Zone immer nur zu mälsiger Entwicklung, so dafs sie manchmal nicht mit wünschenswerter Sicherheit und Schärfe erkannt werden kann. Vortrefflich gelingt aber die Reaktion, wenn man dazu obige eisenhaltige Schwefelsäure und eisenhaltigen Eisessig benützt, welch' letzterer in gleicher Weise wie jene bereitet wird, $d . h$. indem man $100 \mathrm{ccm} E$ is $\theta \mathrm{ssig}$ mit 1 oom der besprochenen Ferrisulfatlosung vermischt.2)

1) Ber. d. pharmac. Gesellschatt 1895, Heft 11. - Keller bringt in der Einleitung seiner Abhandlung eine Zusammenstellung der Resultate Schmiedeberg's, fahrt aber unter diesen - wohl nur in dem hier thel angebrachten Streben, möglichst kurz zu sein - auoh wesent. liche. Ergebnises meiner Arbeiten auf, was ich hiermit berichtigen möchte.

2) Der Eisessig wird hiebei trab und scheidet innerhalb 12 bis 24 Stunden gelblichweilse Flocken ab. Die trübe Lösung ist für den Versuch ebenso gut brauchbar wie die geklärte. 
Zur Ausfuhrung der kombinierten Methode mufs man etwas mehr Substanz nehmen, als bei der einfachen; immerhin genügen auch hier einige zehntel Milligramm.

Löst man Digitoxin in $3-4 \mathrm{ccm}$ des eisenhaltigen Eisessigs und schichtet darunter das gleiche Volumen eisenhaltiger Schwefelsäure, so entsteht sofort an der Grenze beider Flüssigkeiten eine tief dunkle Zone; nach etwa 2 Minuten zeigt sich über dieser ein blauer Streifen; derselbe verbreitert sich langsam und $\mathbf{n}$ a $\mathbf{c h}$ $30 \mathrm{Minuten}$ ruhigen Stehens ist derganze Eisesaig tief indigoblau. Mehrere Stunden später findet sich diese Farbe in ein kräftiges Blaugrün umgewandelt. Die Schwefelsäure färbt sich dagegen so gut wie gar nicht.

Das Digitoxigenin liefert diese prächtige Reaktion nicht; lotztere ist vielmehr ein Charakteristik u m des aus dem Digitoxin gewinnbaren zuckerartigen Spaltungsproduktes, der Digitoxose, welche demnach wohl ebenfalls eine ringtörmige Kohlenstoffkette enthalten dürfte. ${ }^{1}$ )

Digitalinum verum und Digitaligenin färben unter gleichen Bedingungen $n \mathfrak{u}$ die $S \mathrm{ch}$ wefelsäu $r e$ und zwar in gleicher Weise, wie wenn man ohne Eisessig arbeitet.

Hierdurch ist die Möglichkeit gegeben, Digitalinum verum und Digitoxin neben einander zu erkennen; denn ein Gemenge beider Glycoside färbt die Schwefelsäurerotviolettund gleichzeitigden Eisessigindigoblan.

$D i g i t o n i n$ und $D i g i t o g e n$ in ruten auch hierkeinerlei Färbung hervor.

Die aufserordentliche Schärfe, welche speziell der Digitoxinreaktion zukommt, veranla[ste mich sodann, meine frühere Angabe 2) zu kontrollieren, wonach die in ublicher Weise dargestellten Samenglycoside kein Digitoxin enthalten sollen. Thatsächlich ergab die Untersuchung des Digitalinum pur. pulv. germanic. mit eisenhaltigem Eisessig ein völlig negatives Resultat. Wenn Digitoxin in diesem Material vorhanden wäre, ao müfste es sich bei der Darstellung des Digitalinum verum (nach meiner Methode) mit diesem abscheiden

1) In wässriger Lösung giebt die Digitoxose mit Eisenchlorid keine Spur eiuer Färbunc.

2) Dieses Archiv 233, Heft 4. 
oder in dessen Mutterlaugen stecken. Die Trockenrückstände der letzteren ergaben mir keinerlei Digitoxin-Reaktion; ein zufällig vorhandenes Präparat von ro he wigitalinum verum lieterte eine ganz schwache, zweifelhafte Andentung von Blau im Eisessig. Wenn die in üblicher Weise erzengten Samenglycoside also überhanpt Digitoxin enthalten, so kann es sich höchstens um Spuren handeln.

Dagegen verbălt sich ein ans den Blättern gewinnbares Glycosid gegen eisenbaltige Schwefelsäure genan wis das Digitalinum verum. Vorläufig sprechen aber einige andere, gewichtige Grtinde gegen die Identität der beiden Substanzen. Definitive Aufklärung über diesen Punkt sowio uber die Glycoside der Blutter im allgemeinen hoffe ich in kurzer Zeit liefern za können.

Als foststehendeThatsacho kann ich heute echon mitteilen, dalsesnur ein Digitoxingiebt. Schmiedeberg'sund Merck's Praparat haben sich bei genaurer Untersuchung als identisch mit meinem $\beta$-Digitoxin erwiesen, so dals in Zukunft die Präfixa $\alpha$ und $\beta$ zu beseitigen sind.

Schlielslich sei noch darauf hingewiesen, dals die Anwendung der nach obiger Vorschrift bereiteten Schwefelsäure mit wenigstens annähernd bestimmtem Eisengehalte vielleicht noch in manchen anderen Fällen, wo man bisher nur "einen Tropfen Eisenchlorid“ d. h. eine unbestimmte Gröfse anwandte, vorteilhaft sein dürte.

Herrn Dr. M u n k r t danke ich bestens fứr geine Mithilte bei dieser Arbeit. 